\title{
GUT-inspired SUSY and the muon g-2 anomaly: Prospects for LHC $14 \mathrm{TeV}$
}

\author{
Enrico Maria Sessolo* \\ NCBJ, Warsaw \\ E-mail: enrico.sessolo@ncbj.gov.pl
}

\section{Kamila Kowalska}

NCBJ, Warsaw

E-mail: Kamila.kowalska@ncbj.gov.pl

\section{Leszek Roszkowski ${ }^{\dagger}$}

NCBJ, Warsaw

E-mail: L.Roszkowski@sheffield.ac.uk

\author{
Andrew J. Williams \\ NCBJ, Warsaw \\ E-mail: andrew.williamsencbj.gov.pl
}

\begin{abstract}
We consider the possibility that the muon $g-2$ anomaly, $\delta(g-2)_{\mu}$, finds its origins in low energy supersymmetry (SUSY). In the general MSSM the parameter space consistent with $\delta(g-2)_{\mu}$ and correct dark matter relic density of the lightest neutralino easily evades the present direct LHC limits on sparticle masses and also lies to a large extent beyond future LHC sensitivity. The situation is quite different in GUT-defined scenarios where input SUSY parameters are no longer independent. We analyze to what extent the LHC can probe a broad class of GUT-inspired SUSY models with gaugino non-universality that are currently in agreement with the bounds from $\delta(g-2)_{\mu}$, as well as with the relic density and the Higgs mass measurement. To this end we perform a detailed numerical simulation of several searches for electroweakino and slepton production at the LHC and derive projections for the LHC $14 \mathrm{TeV}$ run. We show that, within GUTscale SUSY there is still plenty of room for the explanation of the muon anomaly, although the current LHC data already imply strong limits on the parameter space consistent with $\delta(g-2)_{\mu}$. On the other hand, we demonstrate that the parameter space will be basically fully explored within the sensitivity of the $14 \mathrm{TeV}$ run with $300 \mathrm{fb}^{-1}$. We identify the few surviving spectra that will provide a challenge for detection at the $\mathrm{LHC} 14 \mathrm{TeV}$ run and we characterize their properties.
\end{abstract}

18th International Conference From the Planck Scale to the Electroweak Scale

25-29 May 2015

Ioannina, Greece

*Speaker.

$\dagger$ On Leave of absence from the University of Sheffield, U.K. 


\section{Introduction}

The measurement of the anomalous magnetic moment of the muon, $(g-2)_{\mu}$, shows a $\sim$ $3 \sigma$ discrepancy with the Standard Model (SM) [1]. The measured value of this discrepancy, $\delta(g-2)_{\mu}=a_{\mu}^{\exp }-a_{\mu}^{\mathrm{SM}}$, is

$$
\delta(g-2)_{\mu}=(28.7 \pm 8.0) \times 10^{-10},
$$

where the lowest order hadronic contributions from Ref. [2] are used to compute the SM value.

The discrepancy can easily be accommodated in the Minimal Supersymmetric Standard Model (MSSM), which is rich in particles with the right couplings to provide significant loop contributions to the $\mu \mu \gamma$ vertex. The 1-loop contributions in the MSSM [3] are roughly split into those arising from a chargino/sneutrino loop and those given by smuon/neutralino loops, so that at the lowest order the measurement of $\delta(g-2)_{\mu}$ can be parametrized by

$$
\mu, M_{1}, M_{2}, m_{\tilde{\mu}_{L}}, m_{\tilde{\mu}_{R}}, m_{\tilde{v}_{\mu}}, \tan \beta,
$$

where $\mu$ is the higgsino mass parameter, $\tan \beta$ is the ratio of the Higgs vacuum expectation values (vev's), $M_{1}$ and $M_{2}$ are the bino and wino soft SUSY-breaking masses, $m_{\tilde{\mu}_{L}}$ and $m_{\tilde{\mu}_{R}}$ are smuon soft masses, and $m_{\tilde{v}_{\mu}}$ is the soft mass of the muon sneutrino.

The LHC has started to test the electroweak (EW) sector of the MSSM in a class of searches involving different multiplicities of leptons, no jets, and a significant amount of missing energy [4, $5,6]$. If the experimental limits provided by the CMS and ATLAS collaborations are reinterpreted and applied to general MSSM scenarios the $8 \mathrm{TeV}$ LHC results can only constrain a small part of the available parameter space (see, e.g., [7]), so that ample room remains to attribute a SUSY origin to $\delta(g-2)_{\mu}$.

However, in scenarios where unification of the scalar and gaugino masses is imposed as a remnant of some new physics at the scale of Grand Unification (GUT scale), like in the well-known Constrained MSSM or the Non-Universal Higgs Mass model, it has become virtually impossible to find regions of the parameter space where the measurement of $\delta(g-2)_{\mu}$ can be reproduced (see, e.g., [8]).

Interestingly, this is not necessarily the case for GUT-scale models in which the assumption of gaugino unification is relaxed. It has been pointed out, e.g., in Ref. [9], that in models with GUTdefined values of the gluino soft mass $M_{3} \gg M_{1}, M_{2}$, large $M_{3}$ can drive the masses of colored sparticles to large values at the EW scale through the renormalization group equations (RGEs). Thus one finds agreement with the LHC data and the measurement of the Higgs boson, while at the same time having light sparticles charged under only the EW gauge groups.

In these proceedings we report on our recent paper [10], in which we reinterpreted existing 3-lepton [4, 5] and 2-lepton [6] LHC searches for direct production of charginos, neutralinos, and sleptons, and applied them in combination with constraints from the neutralino relic abundance to the parameter space of several GUT-scale SUSY models that fit $(g-2)_{\mu}$ thanks to non-universal boundary conditions.

We showed that, when the relic density of neutralinos is taken into account, the present constraints from the LHC on the EW sector of this large class of models are already quite stringent 


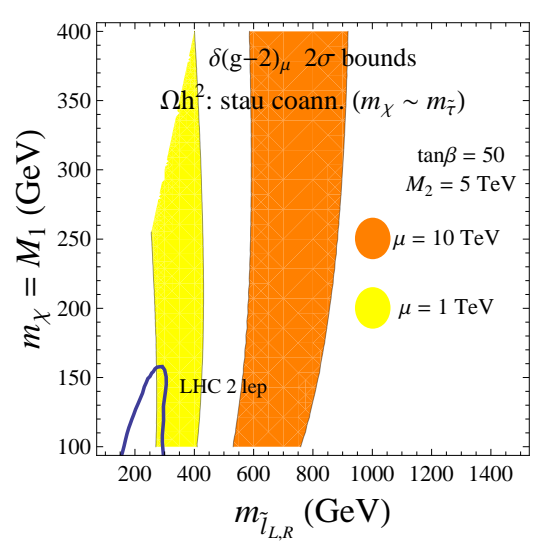

(a)

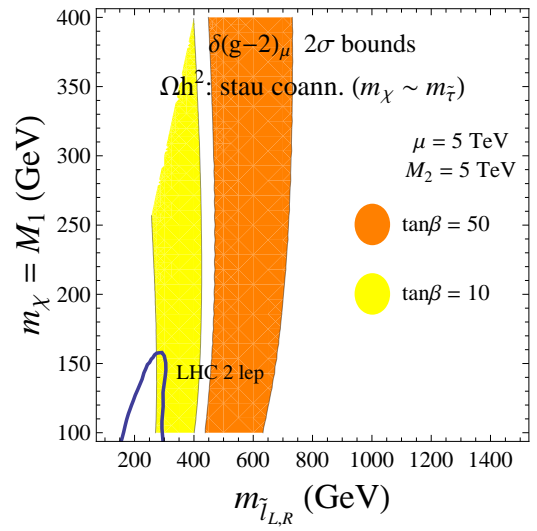

(b)

Figure 1: (a) The parameter space allowed at $2 \sigma$ by $(g-2)_{\mu}$ in the $\left(m_{\tilde{l}_{L, R}}, m_{\chi}\right)$ plane for the stau-coannihilation region of the MSSM. We assume a large value of $\tan \beta$. The orange band shows the case with $\mu=10 \mathrm{TeV}$ and the yellow band the one with $\mu=1 \mathrm{TeV}$. The solid dark blue line shows the approximate 95\% C.L. exclusion bound from 2-lepton searches at the LHC. (b) Same as (a), for a fixed value $\mu=5 \mathrm{TeV}$. The orange band shows the case with $\tan \beta=50$ and the yellow band the one with $\tan \beta=10$.

and, more importantly, that the $14 \mathrm{TeV}$ run offers the opportunity to probe the remaining parameter space virtually in its entirety. To this end, we calculated the projected sensitivity of 2- and 3-lepton searches for the LHC $14 \mathrm{TeV}$ run with $300 \mathrm{fb}^{-1}$ and applied the simulations to the defined GUT-scale models.

\section{The case of the pMSSM: constraints from $(\mathrm{g}-2)_{\mu}$ and the relic density}

We start by reviewing the constraints on the parameter space of the phenomenological MSSM (pMSSM) when the measurement of $\delta(g-2)_{\mu}$ is considered in combination with the relic density. We adopt the simplifying assumptions typical of the pMSSM: the soft SUSY-breaking parameters are here defined at the EW scale and we assume that first and second generation slepton soft masses are degenerate $\left(m_{\tilde{e}_{L}}=m_{\tilde{\mu}_{L}}=m_{\tilde{v}_{e}}=m_{\tilde{v}_{\mu}} \equiv m_{\tilde{l}_{L}}\right.$ and similar identities apply to right-handed sleptons).

To make our point it will be enough to show the case of the stau-coannihilation region, where neutralino/stau coannihilations lead to the measured value of the relic density. Ensuing relations between the mass of the lightest neutralino, $m_{\chi}$, and the other sparticles can be useful in deriving bounds but, as we show below, there remain large fractions of the parameter space outside of the LHC reach. Similar arguments apply to cases in which the correct relic abundance is obtained by other mechanisms: $Z / h$-resonance, coannihilations with charginos, etc. These cases are explicitly analyzed in our paper [10].

In Fig. 1(a) we show the $2 \sigma$ allowed parameter space in the $\left(m_{\tilde{l}_{L, R}}, m_{\chi}\right)$ plane for the staucoannihilation region. The dominant contribution to $\delta(g-2)_{\mu}$ is given in this case by a neutralino/smuon loop, which increases linearly with $\mu$. Thus, very large values of $\mu$ are allowed and they actually help to satisfy the $(g-2)_{\mu}$ constraint. The bounds for two very different values of $\mu$ are shown in the plot, $\mu=1 \mathrm{TeV}$ (yellow band) and $\mu=10 \mathrm{TeV}$ (orange band), while $\tan \beta$ is kept large. 
In Fig. 1(b), the value of $\mu$ is instead fixed at an intermediate value, $\mu=5 \mathrm{TeV}$, and we show the bounds for two different values of $\tan \beta: \tan \beta=10$ (yellow band) and $\tan \beta=50$ (orange band).

We show in both panels the case with $M_{2}=5 \mathrm{TeV}$ and $m_{\tilde{l}_{L}}=m_{\tilde{l}_{R}}$. Note that in the staucoannihilation region the wino soft mass can be actually decoupled and without additional assumptions on the mechanism of SUSY breaking 3-lepton searches are not in principle sensitive to this region of the parameter space. On the other hand, one can see in Figs. 1(a) and 1(b) that the $(g-2)_{\mu}$ constraint bounds the value of the smuon (and selectron) masses, but the sensitivity in 2-lepton searches is still very limited to bite significantly into the parameter space.

This example proves that in this region of the pMSSM one cannot entirely test the hypothesis of a SUSY origin to the $(g-2)_{\mu}$ anomaly at the LHC. This is not only true for the stau-coannihilation region but also for regions characterized by different mechanisms for the neutralino relic density. Thus, in what follows we focus instead on theoretically well-motivated scenarios where the presence of additional symmetries at the GUT scale removes much of the freedom of the parameter space. We limit ourselves to the most common case of a bino-like neutralino, which can saturate the relic density and is testable at the LHC.

\section{GUT-defined models}

Many models with gravity mediation assume for simplicity universal GUT-scale conditions for the gaugino and scalar soft masses. Under those assumptions, however, it has become no longer possible to find parameter space consistent with the $(g-2)_{\mu}$ constraint after the Higgs discovery and null searches for squarks and gluinos at LHC run I. We follow here a different approach and consider GUT-defined models that satisfy the present constraints for $(g-2) \mu$. To this end, we relax the assumption of gaugino universality.

The models we analyze are summarized in Table 1. The scans are subject to a set of constraints, applied through a global likelihood function. The list of constraints, central values, theoretical and experimental uncertainties are presented in Ref. [10].

The first model we consider, Model 1, is a simple modification to the CMSSM, where in addition to the usual parameters, the gluino soft mass $M_{3}$ is allowed to float at the GUT scale, as shown in Table 1. This is the minimal implementation of non-universality that allows one to simultaneously respect the bounds on the color sector from the LHC and the Higgs mass, and those on the EW sector from $(g-2)_{\mu}$. As was shown in Ref. [9], this simple condition can be easily obtained within several GUT symmetries.

In Fig. 2(a) we plot the distribution of the physical left selectron mass, $m_{\tilde{e}_{L}}$, the right selectron mass, $m_{\tilde{e}_{R}}$, and the lightest stau mass, $m_{\tilde{\tau}_{1}}$, versus the neutralino mass, $m_{\chi}$, for the points of Model 1 satisfying our set of constraints at the $2 \sigma$ level. The neutralino is strongly bino-dominated and the correct relic abundance is obtained, for $100 \mathrm{GeV} \lesssim m_{\chi} \lesssim 350 \mathrm{GeV}$, through coannihilation with the lightest stau. For a lighter $m_{\chi}$ it is obtained through bulk-like annihilation to taus via $t$-channel exchange of the moderately light stau. One can see that coannihilation rapidly loses efficiency as $m_{\chi}$ increases, so that no solutions are found for $m_{\chi} \gtrsim 350 \mathrm{GeV}$.

The GUT-scale universality condition is relaxed in Model 2. We show in Fig. 2(b) the distribution of the physical left selectron mass, right selectron mass, and stau mass versus the neutralino 


\begin{tabular}{|c|c|c|}
\hline Model 1 & CMSSM-like $M_{3}$ floating & \\
\hline Parameter & Description & Range \\
\hline$m_{0}$ & Universal scalar mass & 100,4000 \\
\hline$m_{1 / 2}$ & Bino/wino soft mass & 100,4000 \\
\hline$M_{3}$ & Gluino soft mass & 700,10000 \\
\hline$A_{0}$ & Universal trilinear coupling & $-8000,8000$ \\
\hline $\tan \beta$ & Ratio of the Higgs vevs & 2,62 \\
\hline $\operatorname{sgn} \mu$ & Sign of the Higgs/higgsino mass parameter & +1 \\
\hline Model 2 & Non-universal gaugino masses & \\
\hline$M_{1}$ & Bino soft mass & $-4000,4000$ \\
\hline$M_{2}$ & Wino soft mass & $-4000,4000$ \\
\hline$m_{0}, M_{3}, A_{0}, \tan \beta, \operatorname{sgn} \mu$ & Same as Model 1 & Same as Model 1 \\
\hline Model 3 & SO(10)-like sfermions & \\
\hline$m_{16}$ & Universal scalar mass 16 repr. & 100,4000 \\
\hline$m_{10}^{2}$ & Universal scalar mass 10 repr. & $-10000^{2}, 10000^{2}$ \\
\hline $3 M_{D}^{2}$ & $D$-term extra $U(1)$ & $0, m_{16}^{2}-(100 \mathrm{GeV})^{2}$ \\
\hline$m_{1 / 2}$ & Bino/wino soft mass & 100,2000 \\
\hline$M_{3}$ & Gluino soft mass & 800,5000 \\
\hline$A_{0}, \tan \beta, \operatorname{sgn} \mu$ & Same as Model 1 & Same as Model 1 \\
\hline Model 4 & SU(5)-like sfermions & \\
\hline$m_{10}$ & Universal scalar mass 10 repr. & 100,4000 \\
\hline$m_{5}$ & Universal scalar mass $\overline{5}$ repr. & 100,2000 \\
\hline$m_{H_{d}}^{2}$ & Down Higgs doublet soft mass & $-10000^{2}, 10000^{2}$ \\
\hline$m_{H_{u}}^{2}$ & Up Higgs doublet soft mass & $-10000^{2}, 10000^{2}$ \\
\hline$m_{1 / 2}, M_{3}, A_{0}, \tan \beta \operatorname{sgn} \mu$ & Same as Model 3 & Same as Model 3 \\
\hline Model 4-zoom & $\mathbf{S U}(\mathbf{5}) \mu, \mathbf{m}_{\mathbf{A}}$ parametrization & \\
\hline$\mu$ & EW-scale higgsino mass parameter & 10,2000 \\
\hline$m_{A}$ & Pseudoscalar pole mass & 100,4000 \\
\hline$M_{3}$ & Gluino soft mass & 500,2000 \\
\hline$m_{10}, m_{5}, m_{1 / 2}, A_{0}, \tan \beta$ & Same as Model 4 & Same as Model 4 \\
\hline
\end{tabular}

Table 1: Parameters of the models analyzed in this work. All soft SUSY-breaking masses are defined at the GUT scale. Dimensionful quantities are given in $\mathrm{GeV}$ and $\mathrm{GeV}^{2}$.

mass for the $2 \sigma$ allowed points. The mechanism of interest for the relic density is again staucoannihilation, like in Model 1. The main difference with the previous case is that, in addition to a broader range of $m_{\chi_{1}^{ \pm}}$values, there is a broader distribution for the slepton masses of the first two generations, due to the fact that the splitting between the left- and right-handed selectron masses increases with increasing the GUT-scale value of $M_{2}$, as this drives the left-handed mass to larger values through the RGEs.

Model 3 introduces a small difference between the right- and left-handed soft sfermion masses at the GUT-scale. The prototype we have in mind is a supergravity-based, GUT-scale model characterized by $S O(10)$ boundary conditions, where we assume a small positive $D$-term contribution from the extra (broken) $U(1)[11,12]$.

We show in Fig. 3(a) the physical $m_{\tilde{e}_{L}}, m_{\tilde{e}_{R}}$, and $m_{\tilde{\tau}_{1}}$ distributions versus the neutralino mass for the right slepton-coannihilation region. As was the case in Model 1, an upper bound on $m_{\chi}$ can be 


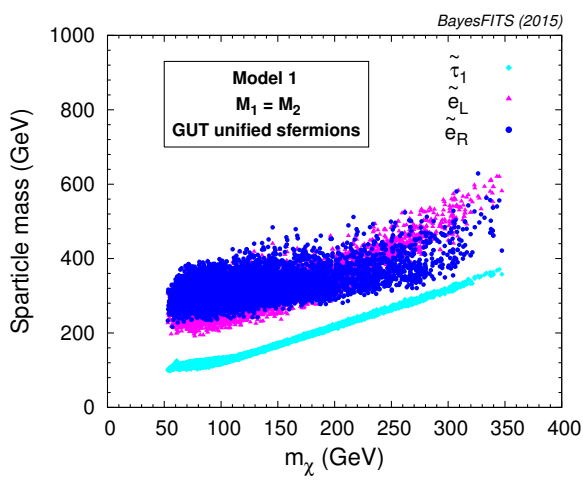

(a)

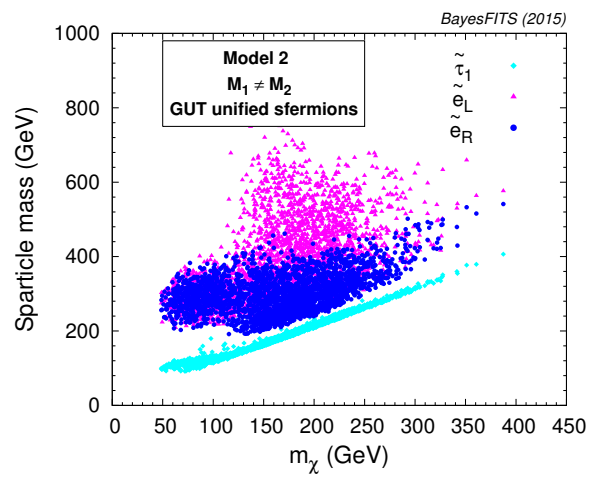

(b)

Figure 2: (a) The distribution of the physical left selectron mass (magenta triangles), right selectron mass (blue circles), and lightest stau mass (cyan diamonds) versus the neutralino mass for the points of Model 1 that satisfy the constraints of Ref. [10] at the $2 \sigma$ level. (b) Same as (a) for Model 2.

derived, $m_{\chi} \lesssim 450 \mathrm{GeV}$, beyond which coannihilation becomes no longer efficient and $\Omega_{\chi} h^{2}$ starts to rise. Figure 3(a) also shows that in this region the left sleptons and, for many points, even the lightest staus, are not much heavier than the neutralino (and the lightest chargino, which is winolike with $m_{\chi_{1}^{ \pm}} \approx 2 m_{\chi}$ ). This has important consequences when it comes to the LHC signatures.

Model 4 extends the allowed GUT-scale ranges for the right- and left-handed sleptons. The prototype we have in mind is a supergravity model with minimal $S U(5)$ boundary conditions at the GUT scale. The GUT-defined soft squark and slepton masses are parametrized in terms of the common scalar soft mass for the fields belonging to the $\overline{\mathbf{5}}$ representation, $m_{5}$, and the common scalar mass in the 10, $m_{10}$. The Higgs doublets' soft masses are free.

Model 4 introduces more freedom to the parameter space, with the consequence that there are

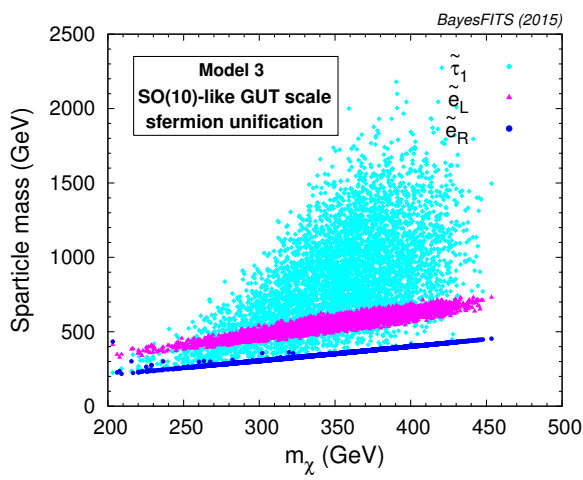

(a)

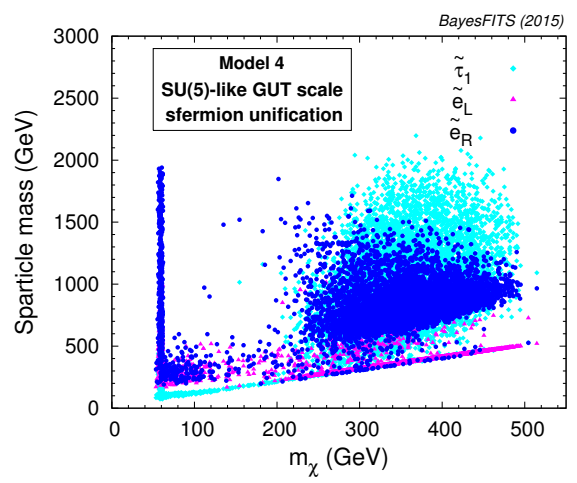

(b)

Figure 3: (a) The distribution of the physical left-handed selectron mass (magenta triangles), right-handed selectron mass (blue circles), and lightest stau mass (cyan diamonds) versus the neutralino mass for the points of Model 3 that are not in common with Model 1. All points satisfy the constraints of Ref. [10] at the $2 \sigma$ level. (b) The distribution of the slepton physical masses in Model 4. 
regions for which the relic density is satisfied thanks to coannihilation of a left slepton of the first or second generation and the neutralino. This is shown in Fig. 3(b), where we plot the physical slepton mass distributions for Model 4.

\section{Constraints and projections for the LHC}

In this section we confront the GUT-defined scenarios discussed in Sec. 3 with the bounds from direct SUSY searches at the LHC.

We numerically reproduce three LHC searches designed to explore the EW sector of the MSSM: the searches for EW chargino and neutralino production with 3 leptons in the final state by ATLAS and CMS $[4,5]$ (collectively called "3-lepton" hereafter), and a search for direct slepton pair production, sneutrino pair production, and slepton/sneutrino production with two opposite-sign leptons in the final state, by ATLAS [6] (dubbed as "2-lepton" hereafter).

Besides the present LHC bounds we also calculate the sensitivity of the ATLAS 2-lepton and CMS 3-lepton searches at the LHC $14 \mathrm{TeV}$ run. We assume $L=300 \mathrm{fb}^{-1}$ integrated luminosity. In each case we simulate the dominant SM backgrounds. For the 3-leptons search these are $W Z$ and $t \bar{t}$ production, as well as rare SM processes such as $t \bar{t} Z / W / H$ and triboson production. For the 2-lepton search the dominant backgrounds come from diboson production and $t \bar{t}$ production.

The efficiencies for the background samples are derived applying the same experimental cuts used for the $8 \mathrm{TeV}$ run, and the number of background events is calculated as the product of the efficiency, luminosity, and cross section. The uncertainty in the number of background events is evaluated as the sum in quadrature of the uncertainty of the cross-section determination and the statistical uncertainty of the efficiency determination with the Monte Carlo simulation. Note that this approach leads to a conservative estimate of future sensitivity, as one expects the eventual uncertainties determined by the experimental collaborations using data-driven methods to be several times smaller.

We show in Fig. 4 the present LHC bounds on the parameter space of the models of Sec. 3. We obtain them by applying the simulation of the searches described above to the model scans. For each point we simulate $10^{5}$ events at LO for each of the relevant production mechanisms: $\tilde{\chi}_{2}^{0} \chi_{1}^{ \pm}$ and $\tilde{\chi}_{1,2}^{ \pm} \tilde{\chi}_{3,4}^{0}$ for the 3-lepton searches and additionally $\tilde{l}_{L}^{+} \tilde{l}_{L}^{-}, \tilde{l}_{R}^{+} \tilde{l}_{R}^{-}, \tilde{l}_{L}^{ \pm} \tilde{v}_{l}, \tilde{v}_{l} \tilde{v}_{l}^{*}$ and $\tilde{\chi}_{1}^{+} \tilde{\chi}_{1}^{-}$for the 2-lepton search. Cross sections are calculated at NLO.

All points satisfy the constraints listed in Ref. [10] at the 95\% C.L. The points excluded at the $95 \%$ C.L. by the 3-lepton searches are shown as light gray triangles, those excluded by the 2-leptons searches as gray circles, and those excluded simultaneously in both topologies as dark gray diamonds. The points that are presently not excluded by the LHC are shown as blue squares.

In Fig. 5 we show the projected 95\% C.L. bounds for the models of Sec. 3. The color code is the same as in Fig. 4. Remarkably, the parameter space can be excluded in its near entirety in all models by the combination of 3-lepton and 2-lepton searches.

The points in the Fig. 5(a) that survive our simulation, shown as blue squares, lie in the compressed spectra region $m_{\tilde{l}_{L}} \approx m_{\chi_{1}^{ \pm}} \simeq 500-600 \mathrm{GeV}$. They also remain beyond the $95 \%$ C.L. reach of the 2-lepton search, although our simulation places them within $68 \%$ C.L. reach. When a more precise background determination is provided by the experimental collaborations, this region may be tested entirely with an even lower luminosity. 


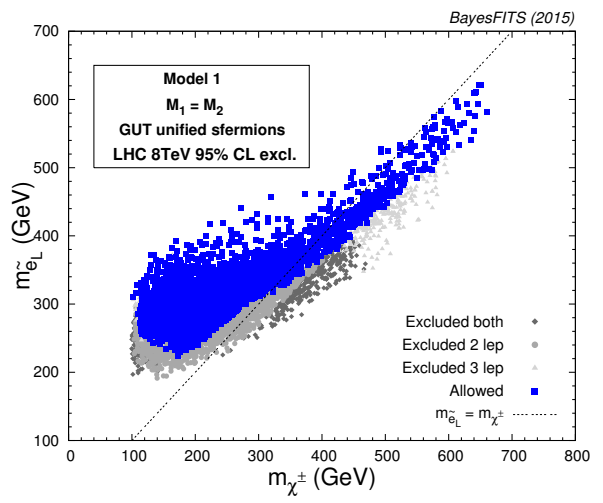

(a)

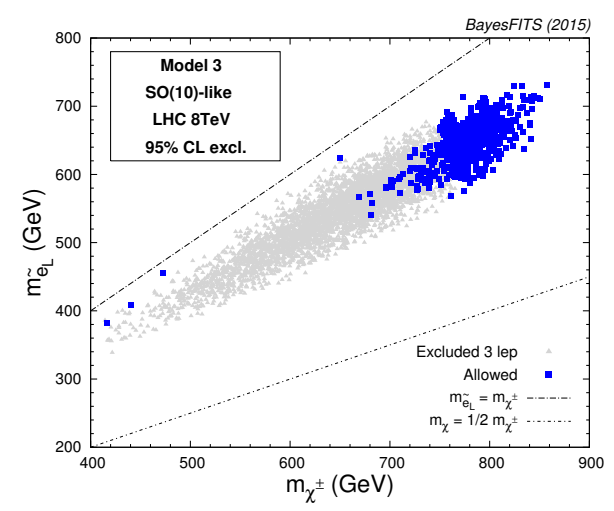

(c)

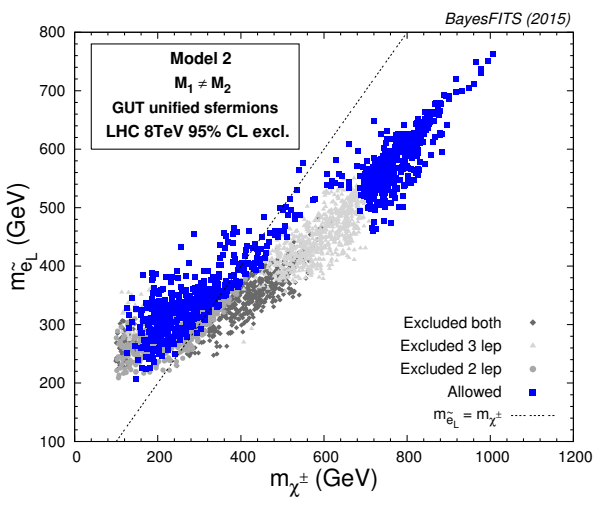

(b)

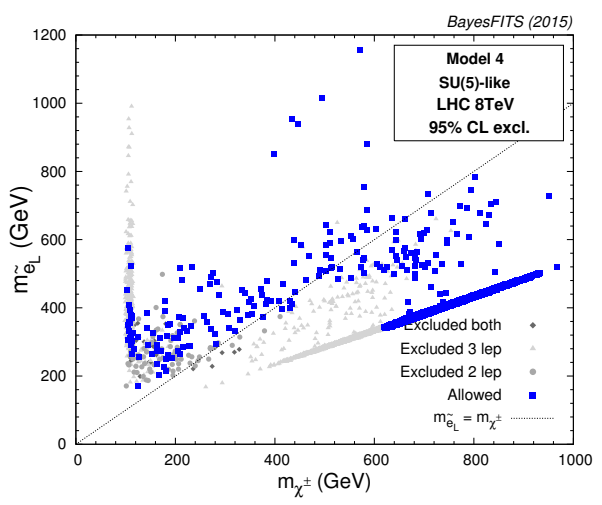

(d)

Figure 4: (a) The bounds from the LHC $8 \mathrm{TeV}$ run on the points of Model 1 in the $\left(m_{\chi_{1}^{ \pm}}, m_{\tilde{e}_{L}}\right)$ plane. Points excluded at the $95 \%$ C.L. by the 3-lepton searches are shown as light gray triangles, points excluded by the 2-lepton search are shown as gray circles, and points excluded in both topologies are shown as dark gray diamonds. Blue squares represent the points still unconstrained at the LHC. (b) Same as (a) for Model 2. (c) Same as (a) for the part of the parameter space of Model 3 that is not common to Model 1. (d) Same as (a) for Model 4.

The points outside of the LHC reach in Fig. 5(b) feature chargino and neutralino masses quite close to each other and they also lie on the compressed region for 2-lepton searches, making this combination of parameters very challenging even for the $14 \mathrm{TeV}$ run.

Figure 5(c) shows that Model 3 entirely falls within reach of the LHC, while Fig. 5(d) shows that Model 4 presents a more interesting situation. In fact, the part of the parameter space that remains unconstrained corresponds to points with $m_{\tilde{e}_{L}}>m_{\chi_{1}^{ \pm}}$, characterized by large branching fractions for $\tilde{\chi}_{2}^{0} \rightarrow \tilde{\tau}_{1} \tau$ or $\tilde{\chi}_{2}^{0} \rightarrow \tilde{\chi}_{1}^{0} h$. The surviving points situated at $m_{\tilde{e}_{L}} \lesssim 600 \mathrm{GeV}$ should be in the future tested by the 2-lepton search even with $L=300 \mathrm{fb}^{-1}$ as the uncertainties in the background will likely be smaller than our estimate. The points with $m_{\tilde{e}_{L}}>600 \mathrm{GeV}$ are characterized by large stau masses, and consequently larger branching fractions to the $\tilde{\chi}_{2}^{0} \chi_{1}^{ \pm} \rightarrow h \tilde{\chi}_{1}^{0} W^{ \pm} \tilde{\chi}_{1}^{0}$ channel. They remain beyond the reach of the 3-lepton search at $300 \mathrm{fb}^{-1}$, but should eventually be tested with $3000 \mathrm{fb}^{-1}$. 


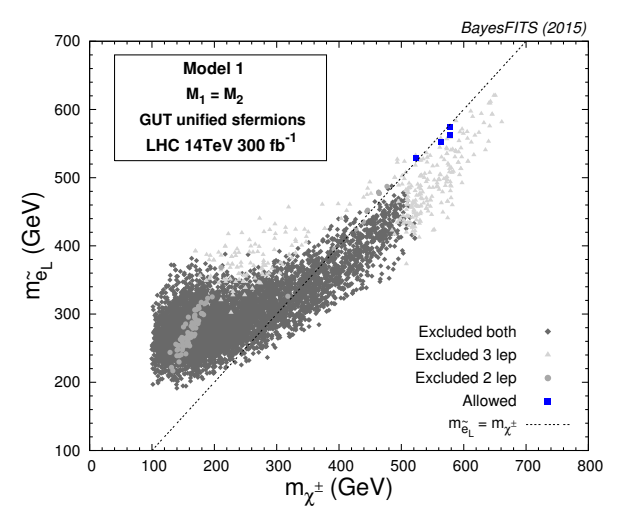

(a)

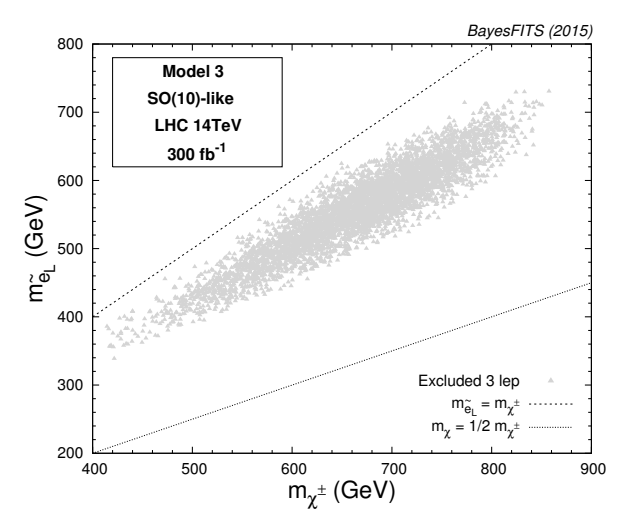

(c)

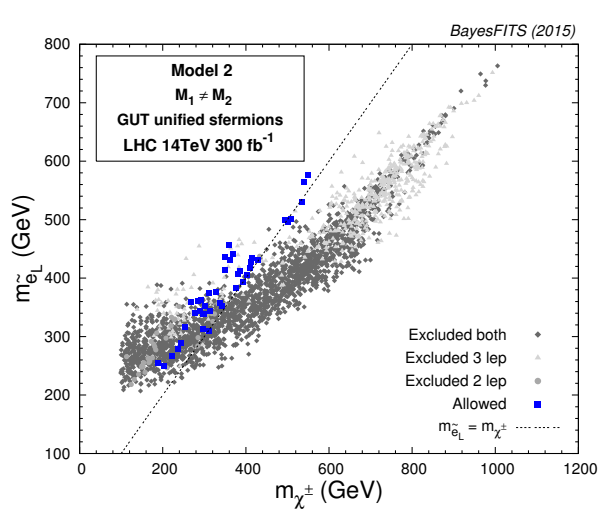

(b)

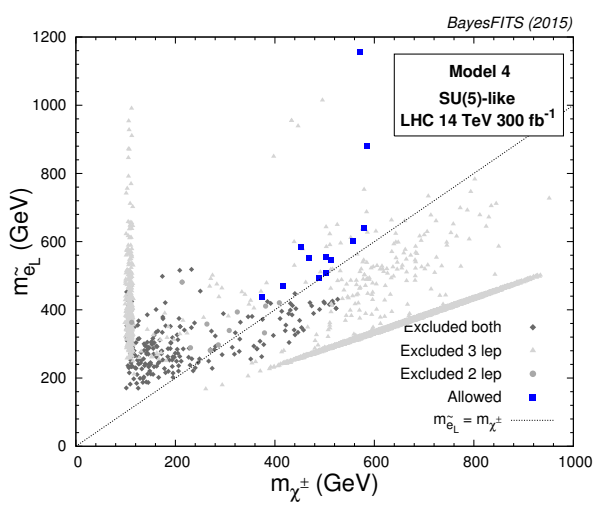

(d)

Figure 5: (a) The expected reach of the LHC $14 \mathrm{TeV}$ run on the points of Model 1 in the $\left(m_{\chi_{1}^{ \pm}}, m_{\tilde{e}_{L}}\right)$ plane. The color code is the same as in Fig. 4. (b) Same as (a) for Model 2. (c) Same as (a) for the part of the parameter space of Model 3 that is not common to Model 1. (d) Same as (a) for Model 4.

\section{Summary}

Our analysis provides strong limits from the $8 \mathrm{TeV}$ run on the parameter space of GUTconstrained scenarios consistent with $(g-2)_{\mu}$. However, a large number of model points are shown to evade the limits, thus leaving ample room for the explanation of the $(g-2)_{\mu}$ anomaly within GUT-scale SUSY models.

We have shown that the parameter space surviving the bounds from the $8 \mathrm{TeV}$ run falls within the sensitivity of the $14 \mathrm{TeV}$ run with $300 \mathrm{fb}^{-1}$ projected luminosity virtually in its entirety. This opens up the interesting possibility that, if the $(g-2)_{\mu}$ anomaly is real and will be confirmed by future dedicated experiments, explanation within a large class of well motivated SUSY models will give sure signatures at the LHC or, alternatively, these models will have to be abandoned as an explanation for the $(g-2)_{\mu}$ anomaly.

A few small regions proved to be difficult to test even at the end of the $14 \mathrm{TeV}$ run, and they should be given special attention:

- Points where the neutralino is bino-like and almost degenerate with the lightest stau. This 
channel is notoriously difficult to test, because of the soft nature of the produced taus.

- Some models can have $M_{2} / M_{1}<1$ at the GUT scale, driving the EW spectrum to be highly compressed even for a predominantly bino-like neutralino.

- In Model 4 the situation can arise where only one slepton is light and almost degenerate with the neutralino. In this case 2-lepton searches will be ineffective and at the same time the sensitivity reach of 3-lepton searches will be curbed by the absence of intermediate, fairly light sleptons. $\tilde{\chi}_{2}^{0}$ thus decays predominantly into the Higgs bosons, requiring a much larger integrated luminosity.

\section{References}

[1] G. W. Bennett et al. [Muon g-2 Collaboration], Final Report of the Muon E821 Anomalous Magnetic Moment Measurement at BNL, Phys. Rev. D 73, 072003 (2006) [hep-ex/0602035].

[2] M. Davier, A. Hoecker, B. Malaescu and Z. Zhang, Reevaluation of the Hadronic Contributions to the Muon g-2 and to alpha(MZ), Eur. Phys. J. C 71, 1515 (2011) [Eur. Phys. J. C 72, 1874 (2012)] [arXiv:1010.4180 [hep-ph]].

[3] T. Moroi, The Muon anomalous magnetic dipole moment in the minimal supersymmetric standard model, Phys. Rev. D 53, 6565 (1996) [Phys. Rev. D 56, 4424 (1997)] [hep-ph/9512396].

[4] G. Aad et al. [ATLAS Collaboration], Search for direct production of charginos and neutralinos in events with three leptons and missing transverse momentum in $\sqrt{s}=8 \mathrm{TeV}$ pp collisions with the ATLAS detector, JHEP 1404, 169 (2014) [arXiv:1402.7029 [hep-ex]].

[5] V. Khachatryan et al. [CMS Collaboration], Searches for electroweak production of charginos, neutralinos, and sleptons decaying to leptons and W, Z, and Higgs bosons in pp collisions at $8 \mathrm{TeV}$, Eur. Phys. J. C 74, no. 9, 3036 (2014) [arXiv:1405.7570 [hep-ex]].

[6] G. Aad et al. [ATLAS Collaboration], Search for direct production of charginos, neutralinos and sleptons in final states with two leptons and missing transverse momentum in pp collisions at $\sqrt{s}=8$ TeV with the ATLAS detector, JHEP 1405, 071 (2014) [arXiv:1403.5294 [hep-ex]].

[7] M. Endo, K. Hamaguchi, S. Iwamoto and T. Yoshinaga, Muon g-2 vs LHC in Supersymmetric Models, JHEP 1401, 123 (2014) [arXiv:1303.4256 [hep-ph]].

[8] A. Fowlie, M. Kazana, K. Kowalska, S. Munir, L. Roszkowski, E. M. Sessolo, S. Trojanowski and Y. L. S. Tsai, The CMSSM Favoring New Territories: The Impact of New LHC Limits and a $125 \mathrm{GeV}$ Higgs, Phys. Rev. D 86, 075010 (2012) [arXiv:1206.0264 [hep-ph]].

[9] S. Akula and P. Nath, Gluino-driven radiative breaking, Higgs boson mass, muon g-2, and the Higgs diphoton decay in supergravity unification, Phys. Rev. D 87, no. 11, 115022 (2013) [arXiv:1304.5526 [hep-ph]].

[10] K. Kowalska, L. Roszkowski, E. M. Sessolo and A. J. Williams, GUT-inspired SUSY and the muon g-2 anomaly: prospects for LHC 14 TeV, JHEP 1506, 020 (2015) [arXiv:1503.08219 [hep-ph]].

[11] Y. Kawamura, H. Murayama and M. Yamaguchi, Low-energy effective Lagrangian in unified theories with nonuniversal supersymmetry breaking terms, Phys. Rev. D 51, 1337 (1995) [hep-ph/9406245].

[12] C. F. Kolda and S. P. Martin, Low-energy supersymmetry with D term contributions to scalar masses, Phys. Rev. D 53, 3871 (1996) [hep-ph/9503445]. 